\title{
Bound states at impurities as a probe of topological superconductivity in nanowires
}

\author{
Jay D. Sau and Eugene Demler \\ Department of Physics, Harvard University, Cambridge, Massachusetts 02138, USA \\ (Received 19 April 2012; revised manuscript received 8 October 2013; published 4 November 2013)
}

\begin{abstract}
Spin-orbit coupled superconductors are interesting candidates for realizing topological and potentially nonAbelian states with Majorana fermions. We argue that time-reversal broken spin-orbit coupled superconductors generically can be characterized as having subgap states that are bound to localized nonmagnetic impurities. Such bound states, which are referred to as the Shiba states, can be detected as sharp resonances in the tunneling spectrum of the spin-orbit coupled superconductors. The Shiba state resonance can be tuned using a gate voltage or a magnetic field from being at the edge of the gap at zero magnetic fields to crossing zero energy when the Zeeman splitting is tuned into the topological superconducting regime. The zero-crossing signifies a fermion parity changing first-order quantum phase transition, which is characterized by a Pfaffian topological invariant. These zero crossings of the impurity level can be used to locally characterize the topological superconducting state in topological nanowires from tunneling experiments.
\end{abstract}

DOI: 10.1103/PhysRevB.88.205402

PACS number(s): 74.78.Na, 73.20.Hb, 73.21.Hb, 74.25.fc

\section{INTRODUCTION}

Majorana fermions (MF) have been the subject of intense recent studies, both due to their fundamental interest as a new type of particle with non-Abelian statistics and their potential application in topological quantum computation (TQC). ${ }^{1-5}$ Topological superconductors ${ }^{6}$ are promising candidates for the practical solid-state realization of MFs. ${ }^{7-15}$ A simple topological superconducting (TS) system supporting MFs, which has attracted considerable experimental attention, ${ }^{2}$ consists of a spin-orbit coupled semiconductor in a magnetic field placed in contact with an ordinary superconductor. ${ }^{1-15}$ In particular, the one-dimensional semiconducting nanowire, can be driven from a conventional or nontopological superconducting (NTS) phase into a TS phase by increasing the magnetic field parallel to the wire above the critical value $V_{Z}>V_{Z, c}=$ $\sqrt{\Delta^{2}+\mu^{2}} \cdot{ }^{14,15}$ Here, $\mu$ is the chemical potential $\mu$ with respect to the bottom of an electron subband of the nanowire and $\Delta$ is the superconducting pairing potential induced from a nearby $s$-wave superconductor. ${ }^{16}$ In fact, recent experimental results ${ }^{17-20}$ on these systems have already shown promising evidence for the existence of MFs.

While experiments such as tunneling and the fractional Josephson effect to detect the presence of end $\mathrm{MFs}^{8,11,14,15,21,22}$ and edge $\mathrm{MFs}^{23-25}$ have been proposed and demonstrated, ${ }^{17,26}$ local probes that characterize the TS state of the wire, away from the physical ends are still missing. Such probes of the TS state of the entire wire segment can provide more reliable characterization of MFs than zero-bias conductance peaks ${ }^{17}$ measured in tunneling, which can also arise from local impurities. In the presence of realistic disorder-induced potential fluctuations, only certain segments of the wire can be expected to be in the TS phase. In this case, local probes of the TS state can allow us to locally determine which segments of a given nanowire are in the TS phase. The zero modes at the end of such segments can be expected to be non-Abelian Majorana modes. The physical ends of the wire, because of the presence of disorder and external potentials might be in a nontopological superconducting phase and therefore might not necessarily support Majorana modes even though some segment in the middle of the wire does.
Scanning tunneling microscopy (STM) of superconductors has provided one route to locally characterizing the superconducting state of unconventional superconductors. ${ }^{27}$ Specifically, superconductors can be characterized by their response to impurities, which can be probed by STM spectroscopy. ${ }^{28,29}$ Magnetic impurities in spin-singlet superconductors lead to Shiba states, which appear as sharp features in tunneling spectra. ${ }^{30}$ Tuning the strength of the impurity has been predicted, in principle, to lead to a local quantum phase transition (QPT) ${ }^{31,32}$ Therefore it is natural to expect that studying the tunneling spectra around impurities in wires might provide a local probe of the superconductivity in the semiconductor nanowire. It has already been argued that the topological superconductivity of $d+i d$ superconductors can be extracted by studying the low-energy bound states associated with magnetic and nonmagnetic impurities. ${ }^{33}$

In this paper, we show that the semiconductor nanowire systems that have been proposed to realize MFs are also ideal for generating subgap Shiba states bound to nonmagnetic impurities. Such Shiba states in semiconductor nanowires in proximity to a superconductor can be probed either by STM or tunnel probe configurations ${ }^{17,34}$ shown in Figs. 1(a) and 2(b). In general, we find that strongly bound Shiba states only occur for large magnetic fields with $V_{Z} \gtrsim \Delta$. Such low-energy impurity bound states are indicative of a cross-over of the superconducting order parameter from an $s$-wave-like state to a $p$-wave-like superconducting state. More specifically, we find that the energy associated with the Shiba state can be tuned to cross zero energy as the impurity strength is increased [see Fig. 1(c)], providing a realization of the fermion-parity changing transition. ${ }^{32}$ Such a zero crossing will be shown to be associated with a local version of the Pfaffian topological invariant that may be used to characterize the TS phase. In practice, the impurity strength might be tuned in the configuration in Fig. 1(b) by varying the gate voltage of a gate which is placed at a distance shorter than the Fermi wavelength (can be $\sim 60 \mathrm{~nm}$ for low-density semiconductors). Gate potentials on length-scales shorter than the Fermi wavelength would effectively induce point-potential scattering in the wire. 

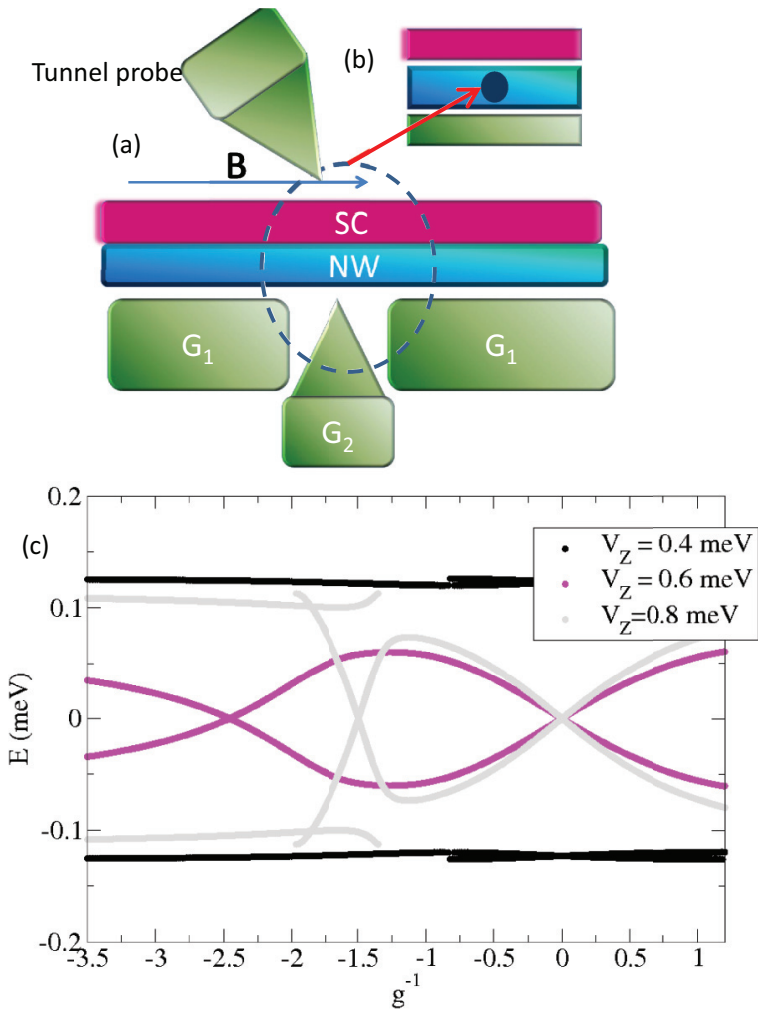

FIG. 1. (Color online) (a) Subgap impurity bound states in a nanowire (NW), in a magnetic field $B$, and in proximity to a superconductor (SC) are localized near a gate-tunable local potential $G_{2}$ and can be detected in tunneling experiments. (b) The gate potential produced by $G_{2}$ can be replaced by a local impurity. (c) Bound-state energy as a function of inverse impurity strength or impurity transparency $g^{-1}$ (in units of $\mathrm{meV}^{-1 / 2}$ ) in the topological phase [i.e., Zeeman potential $V_{Z}=0.6 \mathrm{meV}$ (pink), $V_{Z}=0.8 \mathrm{meV}$ (gray curve)] shows a characteristic zero-energy crossing. The nontopological phase [i.e., for $V_{Z}=0.4 \mathrm{meV}$ (black curve)] shows weakly bound Shiba states.

\section{LOCAL IMPURITY (SHIBA) BOUND STATES IN SUPERCONDUCTORS}

Following the derivation of the spectrum of magnetic impurities in conventional superconductors, ${ }^{30}$ we consider the Green function $G_{\tau \sigma ; \tau^{\prime} \sigma^{\prime}}\left(x ; x^{\prime} ; E\right)$ of the superconducting nanowire in the Nambu spinor notation. Here, $x, x^{\prime}$ represent spatial coordinates on the nanowire, $\sigma, \sigma^{\prime}$ refer to the spin indices and $\tau, \tau^{\prime}=0,1$ represent the particle-hole index, which is needed to describe both the normal and anomalous parts of the Green function of the superconducting nanowire. The Green function $G$ matrix for an impurity in a superconductor can be calculated using the Dyson equation

$$
\begin{aligned}
G\left(x x^{\prime} ; E\right)= & G_{0}\left(x x^{\prime} ; E\right)+\int d x_{1} G^{(0)}\left(x x_{1} ; E\right) \\
& \times V\left(x_{1}\right) G\left(x_{1} x^{\prime} ; E\right),
\end{aligned}
$$

where $V(x) \propto g \delta(x) \tau_{z}$ is the localized impurity potential $\left(V(x) \propto g \sigma_{z} \delta(x)\right.$ for magnetic impurities in conventional superconductors) and $G_{\tau \sigma ; \tau^{\prime} \sigma^{\prime}}^{(0)}\left(x ; x^{\prime} ; E\right)$ is the Green function of a clean superconducting nanowire with a Bogoliubov
de-Gennes (BdG) Hamiltonian

$$
H_{0}(k)=\left(k^{2}-\mu+\alpha \sigma_{y} k\right) \tau_{z}+V_{Z} \sigma_{z}+\Delta \tau_{x},
$$

where $k=i \partial_{x}$ is the wave vector along the wire and $\alpha$ is the strength of Rashba spin-orbit coupling. ${ }^{11,14}$ The matrices $\sigma_{z}$, $\tau_{x}$, and $\tau_{z}$ are Pauli matrices associated with the indices $\sigma$ and $\tau$, respectively. Since $g \delta(x)$ has dimensions of energy, $g$ has dimensions of energy-length. In this work, we are using in units where $\hbar^{2} / 2 m^{*}=1$ (with $m^{*}$ being the effective mass in the semiconductor nanowire), so that the unit of length is $\mathrm{meV}^{-1 / 2}$. Therefore in our work, $g$ has units of $\mathrm{meV}^{1 / 2}$. The BdG Hamiltonian $H_{0}$ can also be used to represent multiband wires $^{35-37}$ if $\mu, V_{Z}, \Delta$, and $g$ are taken to be matrices indexed by the channel index. For local impurity potentials, the energy levels of bound states are determined from the $x=x^{\prime}=0$ part of the Dyson equation (1), which is written as

$$
G(00 ; E)=\left[1-G^{(0)}(00 ; E) V(0)\right]^{-1} G^{(0)}(00 ; E) .
$$

The original Shiba state problem of a magnetic impurity with $V(0)=g \sigma_{z}$ in an $s$-wave superconductor ${ }^{30}$ is solved by studying the poles of Eq. (3), which are given by

$$
\operatorname{Det}\left[g^{-1}-G^{(0)}(00 ; E) \sigma_{z}\right]=0 .
$$

For the conventional $s$-wave superconductor, $V_{Z}=\alpha=0$, so that $\left[H_{0}, \sigma_{z}\right]=0$ and one can solve for states with definite spin $\sigma_{z}= \pm 1$. Using Eq. (2) for the conventional $s$-wave superconductor (i.e., $V_{Z}=\alpha=0, \mu \gg \Delta$ ), the local Green function is calculated to be $G^{(0)}(00 ; E)=\int d k\left[H_{0}(k)-\right.$ $E]^{-1}=\frac{\left(E+\Delta \tau_{x}\right) \rho}{\sqrt{\Delta^{2}-E^{2}}}$, where $\rho$ is the density of states at the Fermi level. Substituting into Eq. (4), we find that the bound-state energy must satisfy $g^{-1}=\sigma_{z} \rho \frac{E \pm \Delta}{\sqrt{\Delta^{2}-E^{2}}}$. The above equation has subgap, i.e., $|E|<\Delta$ solutions for all magnetic impurity strengths $g$, which can even go through $E \sim 0$.

For nonmagnetic impurities for $V_{Z}, \alpha \neq 0$, the Shiba bound state appears as a pole in the Green function $G$ and therefore satisfies the equation

$$
\operatorname{Det}\left[g^{-1}-G^{(0)}(00 ; E) \tau_{z}\right]=0 .
$$

Consistent with Anderson's theorem, ${ }^{36,38}$ Eq. (5) describing subgap states bound to nonmagnetic impurities, is found to have no solutions for $V_{Z}=0$. This continues to hold, even in the presence of a spatially uniform Zeeman potential $V_{Z} \neq 0$, if the spin-orbit coupling $\alpha$ vanishes. Therefore nonmagnetic impurities may lead to localized Shiba bound states in wires only in the presence of a combination of Zeeman splitting and spin-orbit coupling, which are also the ingredients to realize a TS phase.

Numerical solutions of Eq. (5) plotted in Fig. 1 for finite spin-orbit coupling $\alpha$, Zeeman potential $V_{Z}$, show subgap Shiba states bound to even nonmagnetic impurities. While bound Shiba states are found to exist whenever $V_{Z}>0$, one observes that the deeply bound Shiba states with energy significantly below the gap energy (i.e., say $50 \%$ of the gap for definiteness) occur only when $V_{Z} \gtrsim \Delta$. Following Gorkov and Rashba, ${ }^{39}$ the superconducting order parameter that is induced in the nanowire to a combination of $s$-wave and $p$-wave pairings. The $s$-wave pairing component is expected to be destroyed by the Zeeman potential $V_{Z}$ via the ChandrashekharClogston mechanism. ${ }^{40}$ Thus the existence of low-energy 
(a)

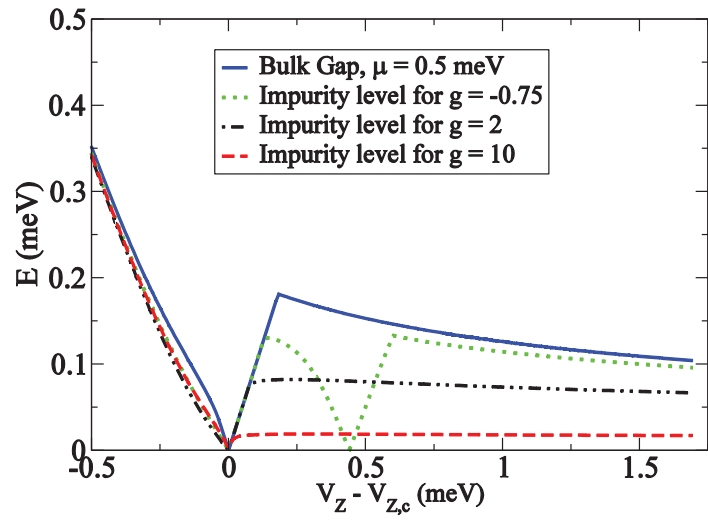

(b)

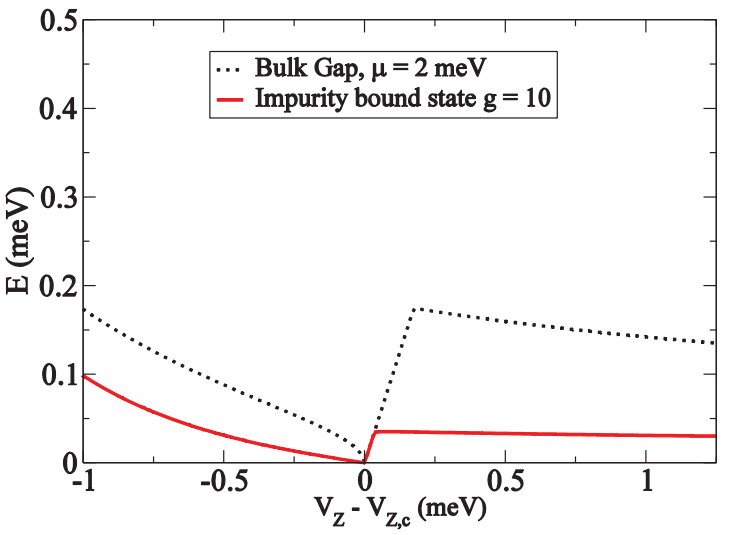

FIG. 2. (Color online) (a) Impurity bound state energy and bulk gap vs Zeeman splitting shows that the topological phase supports deep impurity states significantly (i.e., by a factor of 2) below the gap. (b) For high densities (i.e., $\mu>\Delta$ ), we find similar states even in the nontopological phase for $V_{Z}>\Delta$. Such Shiba bound states might contribute to nearly magnetic field independent low-bias conductance peaks seen in recent experiments. ${ }^{17}$ Here, $\Delta=0.5 \mathrm{meV}$ and the spinorbit coupling $\alpha=0.6$ corresponding to a spin-orbit energy $E_{\mathrm{SO}} \approx$ $50 \mu \mathrm{eV} .{ }^{17}$ The impurity strength $g$ is in units of $\mathrm{meV}^{1 / 2}$.

Shiba states bound to impurities demonstrates a qualitative modification of the superconducting state by the Zeeman field into a $p$-wave pairing phase, which is strongly sensitive to impurities. However, since there is no transition between an $s$-wave dominated superconducting phase and the $p$-wave phase, the effect of the Zeeman field shows up as a crossover between weakly bound and deeply bound Shiba states, which occurs rather sharply near $V_{Z} \sim \Delta$.

\section{SHIBA STATES IN THE TS PHASE}

In contrast to the above crossover, the TS phase is demarcated from the NTS phase by a sharp topological phase transition that can be identified in Fig. 2 by the vanishing of the bulk gap in the wire at $V_{Z}=V_{Z, c}$. The evolution of the Shiba bound state energy as a function of the strength of the impurity $g$ (or magnetic field or density; see discussion below) may be used to precisely distinguish the TS phase from the NTS phase. As seen from Fig. 1, the Shiba bound state energy in the TS phase, in contrast to that in the NTS phase, shows a characteristic pair of crossings of $E=0$ at both zero and nonzero values of the impurity transparency $g^{-1}$. The crossing of the pair of states at $E=0$ is protected by particle-hole symmetry similar to that obtained in the fractional Josephson effect in the TS state. ${ }^{8,9,14}$

One of the $E=0$ crossings in Fig. 1 that occurs at $g^{-1}=0$ can be interpreted as a pair of MFs resulting from splitting the nanowire into two parts. Both strongly attractive or repulsive impurities (i.e., $g^{-1} \rightarrow 0$ ) split the nanowire, since the boundary condition on the wave function $\psi(0) \sim$ $g^{-1}\left[\psi^{\prime}\left(0_{+}\right)-\psi^{\prime}\left(0_{-}\right)\right]$causes $\psi(0) \rightarrow 0$ whenever $|g| \rightarrow \infty$. In the vicitnity of the points $g \rightarrow \pm \infty$, the pair of zero-energy MF split linearly by tunneling as the impurity is tuned away from vanishing transparency in such a way so as to generate a zero-energy crossing of Shiba states at $g^{-1}=0$.

\section{A. Parity-changing QPT}

The zero crossing of the energy of a nondegenerate Shibastate as a function of a parameter such as transparency $g^{-1}$ is accompanied by a change of the fermion-parity of the ground state. To understand this, let us consider the creation operator $d^{\dagger}=\int d x u_{\sigma}(x) \psi_{\sigma}^{\dagger}(x)+v_{\sigma}(x) \psi_{\sigma}(x)$ of a quasiparticle whose $\mathrm{BdG}$ energy eigenvalue is positive for $g^{-1}<g_{0}^{-1}$ and then becomes negative for $g^{-1}>g_{0}^{-1}$. If we define the ground state of the wire to be $|0\rangle$ for $g^{-1}<g_{0}^{-1}$, then $d^{\dagger}|0\rangle$ is an excited state with a higher energy and different fermion parity. As $g^{-1}$ is tuned across the transition so that $g^{-1}>g_{0}^{-1}$, the creation operator $d^{\dagger}$ has a negative energy, which in particular, means that the state $d^{\dagger}|0\rangle$ has a lower energy than the state $|0\rangle$. Therefore the state $d^{\dagger}|0\rangle$, which has a different parity from $|0\rangle$ is the ground state for $g^{-1}>g_{0}^{-1}$, establishing a change in the fermion parity of the ground state at $g^{-1}=g_{0}^{-1}$. The absence of degeneracy such as spin-degeneracy (which is absent because of the large Zeeman potential $V_{Z}$ ) at the crossing at $g^{-1}=g_{0}^{-1}$ is critical to our argument. Technically, when the quasiparticle operator $d^{\dagger}$ approaches zero-energy at $g^{-1}=g_{0}^{-1}$, it is degenerate with the annhilation operator $d$, which has an energy equal in magnitude but opposite in sign to the operator $d^{\dagger}$. However, since the physical states associated with these operators, i.e., $|0\rangle$ and $d^{\dagger}|0\rangle$ have different fermion parities, they cannot hybridize and result in an avoided crossing. The transition discussed here is analogous to the QPT proposed for tunable magnetic impurities in conventional superconductors. ${ }^{32}$ The spin-orbit coupled nanowire provides a realization of this interesting transition using a nonmagnetic impurity, which can be tuned by a local gate voltage. These phase transitions are also analogous to the phase-tuned fermion parity changing transitions in Josephson junctions with magnetic impurities ${ }^{41}$ and also Josephson junctions in topological superconducting systems. ${ }^{8}$

The fermion parity change associated with zero-energy crossings of Shiba states at vanishing impurity transparency $g^{-1} \sim 0$ in the TS phase requires the existence of an odd number of zero-energy crossings at finite impurity transparency $g^{-1} \neq 0$. This follows from the fact that the total number of zero-energy crossings going from $g^{-1}=-\infty$ to $g^{-1}=\infty$ must be even, since both these points are associated with the ground-state Hamiltonian of the nanowire with no impurity, i.e., at $g \sim 0$. Thus the TS phase is characterized by the Shiba bound state energy crossing zero an odd number of times as the limit of infinite impurity strength $g \rightarrow \infty$ is approached from at least one of the sides of either strong repulsive impurities or 
strong attractive impurities. Conversely, since the total number of zero-energy crossings must be even, observing an odd number of zero-energy crossings away from the vanishing transparency point (i.e., $g^{-1}=0$ ) would imply a topologically nontrivial state.

\section{B. Pfaffian associated with local impurity states}

To strengthen the argument that the zero-energy crossing is a topological QPT, we compute the fermion parity using the Pfaffian of the BdG Hamiltonian. ${ }^{8,42}$ The Pfaffian for a particle-hole symmetric $\mathrm{BdG}$ Hamiltonian $H_{\mathrm{BdG}}$ is given by

$$
Q\left(H_{\mathrm{BdG}}\right)=\operatorname{sgn}\left(\operatorname{Pf}\left[H_{\mathrm{BdG}} \Lambda\right]\right),
$$

where the particle-hole matrix $\Lambda=\sigma_{y} \tau_{y}$ transforms creation operators into annihilation operators and vice versa and $H_{\mathrm{BdG}}$ satisfies $H_{\mathrm{BdG}} \Lambda=-\Lambda H_{\mathrm{BdG}}^{*}$. Topological superconducting systems are characterized by a topological invariant, which is defined in terms of the Pfaffian as

$$
F=Q\left(H_{\mathrm{BdG}}(0)\right) Q\left(H_{\mathrm{BdG}}(\pi)\right),
$$

where $H_{\mathrm{BdG}}(0)$ and $H_{\mathrm{BdG}}(\pi)$ are the $\mathrm{BdG}$ Hamiltonians with periodic and antiperiodic boundary conditions for the electrons. Such a definition of the topological invariant, which is related to the change in ground-state fermion parity with boundary conditions, requires the superconductor to be gapped in the bulk. The boundary conditions for the fermions can effectively be changed by introducing a gauge potential along a cut in the system, which for a topological superconducting system will result in the ejection of a fermion along the cut in the system. ${ }^{7}$ For a one-dimensional system, such a cut is a local point defect and therefore the topological invariant for a one-dimensional superconductor can be extracted from the response at a point, as is proposed using the fractional Josephson effect in one dimensional wires. . $^{8,14}$

The present proposal provides an alternative way to change the boundary condition of fermions by changing the strength of a point impurity. Thus a fermion parity changing QPT, which may result from varying the transmitted $\pi$ phase shift, i.e., impurity strength would be a local measure of the topological invariant associated with the superconductor. The assumption of a gapped nature in the bulk allows one to locally determine whether the system is in a topological phase. Despite the fermion parity and the corresponding topological invariant being a global quantity, it is possible to keep track of changes to the fermion parity locally by keeping track of the spectrum at points, such as defects, where fermion energy levels might cross zero energy. As mentioned in the previous section, such zero-energy level crossings are associated with changes in the fermion parity. While the present argument strictly speaking applies to one-dimensional systems, the same argument would apply to rotationally symmetric two-dimensional systems which can be reduced to one-dimensional systems. ${ }^{11}$

Similar to the topological superconductor, the fermionparity changing QPT, can be characterized by a change in the Pfaffian of the BdG Hamiltonian. Since, in an otherwise gapped superconductor, changes to the fermion parity due to a local impurity must be determined locally, it must be possible to characterize the fermion parity change locally. To obtain such a local characterization, we note that zero-energy crossings at an impurity also imply zero-energy crossings of the Green function $U=G^{(0)}(0,0 ; E=0)-g^{-1} \tau_{z}$ [see Eq. (5)]. For a gapped superconductor $G^{(0)}$ and the relevant matrix for determining zero modes $U=G^{(0)}(0,0 ; E=0)-$ $g^{-1} \tau_{z}$ is both Hermitean and particle-hole symmetric (i.e., anticommutes with $\Lambda$ ). Therefore the local Pfaffian invariant is defined as

$$
Q\left(g^{-1}\right)=\operatorname{sgn}\left\{\operatorname{Pf}\left[\left(G^{(0)}(0,0 ; E=0)-g^{-1} \tau_{z}\right) \Lambda\right]\right\} .
$$

The above Pfaffian invariant can only change sign when the determinant of $U=G^{(0)}(0,0 ; E=0)-g^{-1} \tau_{z}$ vanishes, which corresponds to a zero crossing of a Shiba bound-state energy since such a crossing is a solution of Eq. (5) at $E=0$. The fermion parity changing transition of the ground state as a function of impurity strength $g$ is characterized by the topological invariant $Q\left(g^{-1}\right)$ changing sign. Therefore the local fermion parity at the impurity can be calculated directly from computing the local fermion parity $Q\left(g^{-1}\right)$ without computing the Shiba bound states.

\section{Variation of impurity states with Zeeman potential}

In addition to varying the strength of electrostatically induced impurities in a wire, which can, in principle, be tuned in a simpler way than magnetic impurities required for $s$-wave superconductors, ${ }^{32}$ one can also tune the chemical potential or the magnetic field towards the gap-closing topological phase transition to vary the effective impurity strength. A typical impurity can be expected to have a stronger effect as one approaches the phase transition where the quasiparticle gap closes effectively varying the impurity strength. However, this cannot directly be used to characterize the TS phase, since it is difficult to ensure that the effective impurity strength can be varied over the entire range. The evolution of the Shiba states across the phase transition for a fixed impurity strength, which is plotted in Fig. 3, shows a zero-energy crossing of a

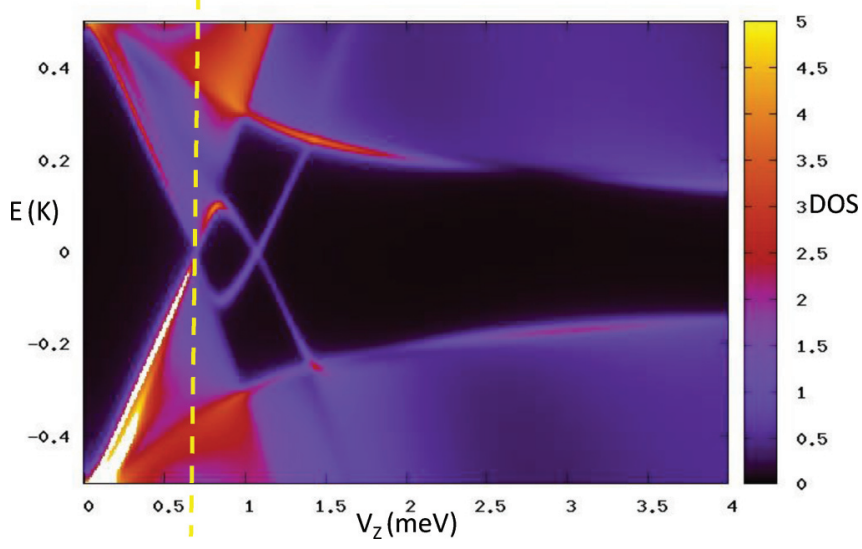

FIG. 3. (Color online) The evolution of the tunneling DOS associated with Shiba bound states with the Zeeman potential $V_{Z}$ also shows zero-energy crossings in the topological superconducting phase (i.e., right of the dashed yellow line) of the nanowire. Here, $\mu=0.2 \mathrm{meV}, \Delta=0.5 \mathrm{meV}$, and $g=2.0 \mathrm{meV}^{1 / 2}$. The apparent zero-energy crossing near the phase transition (dashed yellow line) separating the topological and nontopological phases is excluded from the count because the localized Shiba state does not exist at this gapless point. 
state in the TS phase, similar to the zero-energy crossings as a function of impurity strength plotted in Fig. 1. The result is expected to hold for an impurity that is sufficiently weak away from the phase transition. As the Zeeman splitting from the magnetic field approaches $V_{Z, c}$, the effective strength of the impurity increases relative to the gap leading to an odd number of crossings.

\section{CONCLUSION}

Similar to magnetic impurities, $s$-wave superconductors, ${ }^{28}$ and nonmagnetic impurities in unconventional superconductors, ${ }^{27}$ the Shiba bound states can be used to characterize the superconducting phases of the nanowire. Specifically, we find that in the nanowire the Shiba bound states crossover from weakly bound states to deeply bound states as the magnetic field is increased, indicating a crossover from an $s$-wave-like state for $V_{Z} \lesssim \Delta$ to a $p$-wave-like phase for $V_{Z} \gtrsim$ $\Delta$. Such low-energy Shiba states for $V_{Z} \gtrsim \Delta$ would appear as low-energy conductance features above a critical Zeeman potential $V_{Z}>\Delta$, which would be qualitatively similar to the magnetic field dependence measured in recent experiments ${ }^{17}$ and might provide a possible alternative explanation for these experiments. More importantly, we find that the TS phase is sharply characterized by the presence of an odd number of zero-energy crossings of the impurity bound state both as a function of impurity strength of Zeeman energy. Therefore the tunneling characterization of impurities in the middle of the semiconductor nanowire should provide convincing evidence for the existence of a topological phase in these wires.

Note added. Recently, we became aware of a related work ${ }^{43}$ that predicts low-energy states in the limit of strong impurities. In the context of our work, this corresponds to one of the zero crossings at $g^{-1}=0$ in Fig. 1(c).

\section{ACKNOWLEDGMENTS}

We acknowledge valuable discussions with Anton Akhmerov, Shou-Cheng Zhang, and Ali Yazdani in the course of this work. J.S. thanks the Harvard Quantum Optics Center for support. E.D. acknowledges support from Harvard-MIT CUA, NSF Grant No. DMR-07-05472.
${ }^{1}$ C. Nayak, S. H. Simon, A. Stern, M. Freedman, and S. Das Sarma, Rev. Mod. Phys. 80, 1083 (2008).

${ }^{2}$ F. Wilczek, Nat. Phys. 5, 614 (2009); B. Goss-Levi, Phys. Today 64, 20 (2011); A. Stern, Nature (London) 464, 187 (2010).

${ }^{3}$ A. Kitaev, Ann. Phys. 303, 2 (2003).

${ }^{4}$ G. Moore and N. Read, Nucl. Phys. B 360, 362 (1991).

${ }^{5}$ F. Wilczek, Fractional Statistics and Anyon Superconductivity (World Scientific, Singapore, 1990).

${ }^{6}$ A. P. Schnyder, S. Ryu, A. Furusaki, and A. W. W. Ludwig, Phys. Rev. B 78, 195125 (2008).

${ }^{7}$ N. Read and D. Green, Phys. Rev. B 61, 10267 (2000).

${ }^{8}$ A. Y. Kitaev, Phys. Usp. 44, 131 (2001).

${ }^{9}$ L. Fu and C. L. Kane, Phys. Rev. Lett. 100, 096407 (2008).

${ }^{10}$ C. Zhang, S. Tewari, R. M. Lutchyn, and S. Das Sarma, Phys. Rev. Lett. 101, 160401 (2008); M. Sato, Y. Takahashi, and S. Fujimoto, ibid. 103, 020401 (2009).

${ }^{11}$ J. D. Sau, R. M. Lutchyn, S. Tewari, and S. Das Sarma, Phys. Rev. Lett. 104, 040502 (2010).

${ }^{12}$ J. Alicea, Phys. Rev. B 81, 125318 (2010).

${ }^{13}$ J. D. Sau, S. Tewari, R. M. Lutchyn, T. D. Stanescu, and S. Das Sarma, Phys. Rev. B 82, 214509 (2010).

${ }^{14}$ R. M. Lutchyn, J. D. Sau, and S. Das Sarma, Phys. Rev. Lett. 105, 077001 (2010).

${ }^{15}$ Y. Oreg, G. Refael, and F. von Oppen, Phys. Rev. Lett. 105, 177002 (2010).

${ }^{16}$ J. D. Sau, R. M. Lutchyn, S. Tewari, and S. Das Sarma, Phys. Rev. B 82, 094522 (2010).

${ }^{17}$ V. Mourik, K. Zuo, S. M. Frolov, S. R. Plissard, E. P. A. M. Bakkers, and L. P. Kouwenhoven, Science 336, 1003 (2012).

${ }^{18}$ M. T. Deng, C. L. Yu, G. Y. Huang, M. Larsson, P. Caroff, and H. Q. $\mathrm{Xu}$, Nano Lett. 12, 6414 (2012).

${ }^{19}$ A. Das, Y. Ronen, Y. Most, Y. Oreg, M. Heiblum, and H. Shtrikman, Nat. Phys. 8, 887 (2012).

${ }^{20} \mathrm{C}$. M. Marcus (private communication).
${ }^{21}$ K. Sengupta, I. Zutic, H.-J. Kwon, V. M. Yakovenko, and S. Das Sarma, Phys. Rev. B 63, 144531 (2001).

${ }^{22}$ H. Kwon, K. Sengupta, and V. M. Yakovenko, Low Temp. Phys. 30, 613 (2004).

${ }^{23}$ K. T. Law, P. A. Lee, and T. K. Ng, Phys. Rev. Lett. 103, 237001 (2009).

${ }^{24}$ P. A. Ioselevich and M. V. Feigelman, Phys. Rev. Lett. 106, 077003 (2011).

${ }^{25}$ A. L. Rakhmanov, A. V. Rozhkov, and Franco Nori, Phys. Rev. B 84, 075141 (2011)

${ }^{26}$ L. P. Rokhinson, X. Liu, and J. K. Furdyna, Nat. Phys. 8, 795 (2012).

${ }^{27}$ A. V. Balatsky, I. Vekhter, and J.-X. Zhu, Rev. Mod. Phys. 78, 373 (2006); D. Podolsky, E. Demler, K. Damle, and B. I. Halperin, Phys. Rev. B 67, 094514 (2003); J. M. Byers, M. E. Flatté, and D. J. Scalapino, Phys. Rev. Lett. 71, 3363 (1993); M. I. Salkola, A. V. Balatsky, and D. J. Scalapino, ibid. 77, 1841 (1996); J. E. Hoffman, K. McElroy, D.-H. Lee, K. M. Lang, H. Eisaki, S. Uchida, and J. C. Davis, Science 297, 1148 (2002); Q.-H. Wang and D.-H. Lee, Phys. Rev. B 67, 020511 (2003); H.-D. Chen, J.-P. Hu, S. Capponi, E. Arrigoni, and S.-C. Zhang, Phys. Rev. Lett. 89, 137004 (2002); M. Franz, D. E. Sheehy, and Z. Tesanovic, ibid. 88, 257005 (2002).

${ }^{28}$ A. Yazdani, B. A. Jones, C. P. Lutz, M. F. Crommie, and D. M. Eigler, Science 275, 1767 (1997).

${ }^{29}$ S.-H. Ji, T. Zhang, Y.-S. Fu, X. Chen, J.-F. Jia, Q.-K. Xue, and X-C. Ma, Appl. Phys. Lett. 96, 073113 (2010).

${ }^{30}$ H. Shiba, Prog. Theor. Phys. 40, 435 (1968).

${ }^{31}$ A. Sakurai, Prog. Theor. Phys. 44, 1472 (1970).

${ }^{32}$ M. I. Salkola, A. V. Balatsky, and J. R. Schrieffer, Phys. Rev. B 55, 12648 (1997); R. Vakhitov, S. N. Artemenko, S. V. Remizov, JETP 111, 304 (2010).

${ }^{33}$ F. Wang, Q. Liu, T. Ma, and X. Jiang, J. Phys.: Condens. Matter 24, 455701 (2012). 
${ }^{34}$ E. J. H. Lee, X. Jiang, R. Aguado, G. Katsaros, C. M. Lieber, and S. De Franceschi, Phys. Rev. Lett. 109, 186802 (2012); J-D. Pillet, C. H. L. Quay, P. Morfin, C. Bena, A. Levy Yeyati, and P. Joyez, Nat. Phys. 6, 965 (2010).

${ }^{35}$ M. Wimmer, A. R. Akhmerov, M. V. Medvedyeva, J. Tworzydlo, and C. W. J. Beenakker, Phys. Rev. Lett. 105, 046803 (2010).

${ }^{36}$ A. C. Potter and P. A. Lee, Phys. Rev. Lett. 105, 227003 (2010).

${ }^{37}$ R. M. Lutchyn, T. D. Stanescu, and S. Das Sarma, Phys. Rev. Lett. 106, 127001 (2011).

${ }^{38}$ P. W. Anderson, J. Phys. Chem. Solids 11, 26 (1959).
${ }^{39}$ L. P. Gor'kov and E. I. Rashba, Phys. Rev. Lett. 87, 037004 (2001).

${ }^{40}$ B. S. Chandrasekhar, Appl. Phys. Lett. 1, 7 (1962); A. M. Clogston, Phys. Rev. Lett. 9, 266 (1962).

${ }^{41}$ A. Martin-Rodero and A. L. Yeyati, Adv. Phys. 60, 899 (2011).

${ }^{42}$ P. Ghosh, J. D. Sau, S. Tewari, and S. Das Sarma, Phys. Rev. B 82, 184525 (2010).

${ }^{43}$ H. Hu, L. Jiang, H. Pu, Y. Chen, and X-J. Liu, Phys. Rev. Lett. 110, 020401 (2013). 